\title{
Search for lightly ionizing particles with the MACRO detector
}

M. Ambrosio, ${ }^{12}$ R. Antolini, ${ }^{7}$ G. Auriemma, ${ }^{14, a}$ D. Bakari,${ }^{2,17}$ A. Baldini, ${ }^{13}$ G. C. Barbarino, ${ }^{12}$ B. C. Barish, ${ }^{4}$ G. Battistoni ${ }^{6, b}$ R. Bellotti, ${ }^{1}$ C. Bemporad ${ }^{13}$ P. Bernardini, ${ }^{10}$ H. Bilokon, ${ }^{6}$ V. Bisi,${ }^{16}$ C. Bloise,${ }^{6}$ C. Bower,${ }^{8}$ M. Brigida, ${ }^{1}$

S. Bussino, ${ }^{18}$ F. Cafagna, ${ }^{1}$ M. Calicchio, ${ }^{1}$ D. Campana,${ }^{12}$ M. Carboni, ${ }^{6}$ S. Cecchini, ${ }^{2, c}$ F. Cei, ${ }^{11,13}$ V. Chiarella, ${ }^{6}$ B. C. Choudhary, ${ }^{4}$ S. Coutu, ${ }^{11, j}$ G. De Cataldo, ${ }^{1}$ H. Dekhissi, ${ }^{2,17}$ C. De Marzo, ${ }^{1}$ I. De Mitri, ${ }^{10}$ J. Derkaoui, ${ }^{2,17}$ M. De Vincenzi ${ }_{1}^{18}$ A. Di Credico, ${ }^{7}$ O. Erriquez, ${ }^{1}$ C. Favuzzi, ${ }^{1}$ C. Forti, ${ }^{6}$ P. Fusco, ${ }^{1}$ G. Giacomelli, ${ }^{2}$ G. Giannini, ${ }^{13, e}$ N. Giglietto, ${ }^{1}$ M. Giorgini, ${ }^{2}$ M. Grassi ${ }_{13}^{13}$ L. Gray, ${ }^{7}$ A. Grillo, ${ }^{7}$ F. Guarino, ${ }^{12}$ C. Gustavino, ${ }^{7}$ A. Habig, ${ }^{3}$ K. Hanson, ${ }^{11}$ R. Heinz, ${ }^{8}$ E. Iarocci, ${ }^{6, f}$ E. Katsavounidis, ${ }^{4}$ I. Katsavounidis, ${ }^{4}$ E. Kearns ${ }^{3}{ }^{3}$ H. Kim, ${ }^{4}$ S. Kyriazopoulou, ${ }^{4}$ E. Lamanna, ${ }^{14,1}$ C. Lane, ${ }^{5}$ D. S. Levin, ${ }^{11}$ P. Lipari, ${ }^{14}$ N. P. Longley, ${ }^{4, i}$ M. J. Longo, ${ }^{11}$ F. Loparco, ${ }^{1}$ F. Maaroufi, ${ }^{2,17}$ G. Mancarella, ${ }^{10}$ G. Mandrioli, ${ }^{2}$ A. Margiotta, ${ }^{2}$ A. Marini,${ }^{6}$ D. Martello,${ }^{10}$ A. Marzari-Chiesa, ${ }^{16}$ M. N. Mazziotta, ${ }^{1}$ D. G. Michael, ${ }^{4}$ S. Mikheyev,,${ }^{4, g}$ L. Miller, ${ }^{8, k}$ P. Monacelli, ${ }^{9}$ T. Montaruli, ${ }^{1}$ M. Monteno, ${ }^{16}$ S. Mufson, ${ }^{8}$ J. Musser, ${ }^{8}$ D. Nicolò, ${ }^{13, d}$ R. Nolty,${ }^{4}$ C. Orth, ${ }^{3}$ C. Okada, ${ }^{3}$ G. Osteria, ${ }^{12}$ M. Ouchrif,${ }^{2,17}$ O. Palamara, ${ }^{7}$ V. Patera,${ }^{6, f}$ L. Patriziii, ${ }^{2}$ R. Pazzi,${ }^{13}$ C. W. Peck,${ }^{4}$ L. Perrone,${ }^{10}$ S. Petrera, ${ }^{9}$ P. Pistilli, ${ }^{18}$ V. Popa ${ }^{2, \mathrm{~h}}$ A. Rainò, ${ }^{1}$ J. Reynoldson, ${ }^{7}$ F. Ronga, ${ }^{6}$ C. Satriano, ${ }^{14, a}$ L. Satta, ${ }^{6, f}$ E. Scapparone, ${ }^{7}$ K. Scholberg, ${ }^{3}$ A. Sciubba, ${ }^{6,}$ P. Serra, ${ }^{2}$ M. Sioli, ${ }^{2}$ M. Sitta, ${ }^{16}$ P. Spinelli, ${ }^{1}$ M. Spinetti, ${ }^{6}$ M. Spurio,${ }^{2}$ R. Steinberg, ${ }^{5}$ J. L. Stone,${ }^{3}$ L. R. Sulak, ${ }^{3}$ A. Surdo, ${ }^{10}$ G. Tarlè, ${ }^{11}$ V. Togo,${ }^{2}$ M. Vakili, ${ }^{15}$ E. Vilela, ${ }^{2}$ C. W. Walter,,${ }^{3,4}$ and R. Webb ${ }^{15}$

\author{
(MACRO Collaboration) \\ ${ }^{1}$ Dipartimento di Fisica dell'Università di Bari and INFN, 70126 Bari, Italy \\ ${ }^{2}$ Dipartimento di Fisica dell'Università di Bologna and INFN, 40126 Bologna, Italy \\ ${ }^{3}$ Physics Department, Boston University, Boston, Massachusettes 02215 \\ ${ }^{4}$ California Institute of Technology, Pasadena, California 91125 \\ ${ }^{5}$ Department of Physics, Drexel University, Philadelphia, Pennsylvania 19104 \\ ${ }^{6}$ Laboratori Nazionali di Frascati dell'INFN, 00044 Frascati Roma, Italy \\ ${ }^{7}$ Laboratori Nazionali del Gran Sasso dell'INFN, 67010 Assergi L'Aquila, Italy \\ ${ }^{8}$ Departments of Physics and of Astronomy, Indiana University, Bloomington, Indiana 47405 \\ ${ }^{9}$ Dipartimento di Fisica dell'Università dell'Aquila and INFN, 67100 L'Aquila, Italy \\ ${ }^{10}$ Dipartimento di Fisica dell'Università di Lecce and INFN, 73100 Lecce, Italy \\ ${ }^{11}$ Department of Physics, University of Michigan, Ann Arbor, Michigan 48109 \\ ${ }^{12}$ Dipartimento di Fisica dell'Università di Napoli and INFN, 80125 Napoli, Italy \\ ${ }^{13}$ Dipartimento di Fisica dell'Università di Pisa and INFN, 56010 Pisa, Italy \\ ${ }^{14}$ Dipartimento di Fisica dell'Università di Roma 'La Sapienza"' and INFN, 00185 Roma, Italy \\ ${ }^{15}$ Physics Department, Texas A\&M University, College Station, Texas 77843 \\ ${ }^{16}$ Dipartimento di Fisica Sperimentale dell'Università di Torino and INFN, 10125 Torino, Italy \\ ${ }^{17}$ L.P.T.P., Faculty of Sciences, University Mohamed I, B.P. 524 Oujda, Morocco \\ ${ }^{18}$ Dipartimento di Fisica dell'Università di Roma Tre and INFN Sezione Roma Tre, 00146 Roma, Italy
}

(Received 14 February 2000; published 4 August 2000)

\begin{abstract}
A search for lightly ionizing particles has been performed with the MACRO detector. This search was sensitive to particles with charges between $\frac{1}{5} e$ and close to the charge of an electron, with $\beta$ between approximately 0.25 and 1.0. Unlike previous searches both single track events and tracks buried within high multiplicity muon showers were examined. In a period of approximately one year no candidates were observed. Assuming an isotropic flux, for the single track sample this corresponds to a $90 \%$ C.L. upper flux limit $\Phi$ $\leqslant 9.2 \times 10^{-15} \mathrm{~cm}^{-2} \mathrm{~s}^{-1} \mathrm{sr}^{-1}$.

PACS number(s): $14.80 .-\mathrm{j}, 96.40 .-\mathrm{z}$
\end{abstract}

\footnotetext{
${ }^{a}$ Also at Università della Basilicata, 85100 Potenza, Italy.

${ }^{\mathrm{b}}$ Also at INFN Milano, 20133 Milano, Italy.

${ }^{\mathrm{c}}$ Also at Instituto TESRE/CNR, 40129 Bologna, Italy.

${ }^{\mathrm{d} A l s o}$ at Scuola Normale Superiore di Pisa, 56010 Pisa, Italy.

${ }^{\mathrm{e}}$ Also at Università di Trieste and INFN, 34100 Trieste, Italy.

${ }^{\mathrm{f}}$ Also at Dipartimento di Energetica, Università di Roma, 00185 Roma, Italy.

${ }^{\mathrm{g}}$ Also at Institute for Nuclear Research, Russian Academy of Science, 117312 Moscow, Russia.

${ }^{\mathrm{h}}$ Also at Institute for Space Sciences, 76900 Bucharest, Romania.

${ }^{\mathrm{i}}$ The Colorado College, Colorado Springs, CO 80903.

${ }^{\mathrm{j} A l s o}$ at Department of Physics, Pennsylvania State University, University Park, PA 16801.

${ }^{\mathrm{k}}$ Also at Department of Physics, James Madison University, Harrisonburg, VA 22807.

${ }^{1}$ Also at Dipartimento di Fisica dell'Università della Calabria, Rende Cosenza, Italy.
} 


\section{INTRODUCTION}

Ever since Millikan's historic experiment determined that the charge on matter comes in discrete units [1], experimenters have spent much time and effort first determining the precise value of that charge, and later trying to observe instances in nature where anything other than an integer multiple version of that charge exists.

The first hint that such objects might be present in nature were the results obtained from the deep inelastic scattering experiments at SLAC during the late 1960s [2]. These experiments first demonstrated that nucleons do in fact have substructure. By exploring the structure functions in these scattering experiments, it was discovered that protons and neutrons were constructed of smaller pointlike partons, and that there were three charge-bearing partons in each of the proton and the neutron [3].

This observed parton structure fit well into the quark model previously proposed by Gell-Man and Zweig [4-6]. Although in this model the quarks which make up the baryons and mesons have fractional charge, they are always combined in a way that results in an integrally charged baryon or meson.

Despite decades of searching no one has yet observed a quark free of its ever-present neighbors. Also, the search for electrons or other leptonic-type particles with fractional charge has been in vain. These include larger and more sophisticated versions of Millikan's oil drop experiment, searches in bulk matter, experiments at accelerators, and searches in the cosmic radiation [7-11]. A clear observation of fractional charge would be extremely important since, depending on the type of particle seen, it might mean that confinement breaks down under some circumstances or that entirely new classes of particles exist.

In grand unified theories it is relatively easy to accommodate fractional charge in color singlets by extending the unification group from $\mathbf{S U}(\mathbf{5})$ to a larger group. For example, an extension to $\mathbf{S U}(7)$ allows for charges of $\frac{1}{3}$ [12], another which allows $\frac{1}{3} e$ charge leptons has gauge group SU(5) $\times \mathbf{S U}(\mathbf{5})^{\prime}[13]$. Other grand unified theory groups have been considered which allow for fractional charge, including SU (8) [14], SO (14) [15], and SO (18) [16]. Further, some theories of spontaneously broken QCD have also predicted free quarks [17], although these quarks would probably be contained in super-heavy quark-nucleus complexes with large nonintegral charge.

This paper presents the results of a search for penetrating, weakly interacting particles with fractional charge in the cosmic radiation with the MACRO detector. A more detailed description of this analysis can be found in [18]. Since a particle of charge $Q$ has a rate of energy loss by atomic excitation and ionization proportional to $Q^{2}$, particles of a given velocity with fractional charge deposit less energy in a detector than particles with unit charge. So, for example, a particle traveling at relativistic speed with charge of $\frac{1}{3} e$ will have an energy deposition only $\frac{1}{9}$ that of the muon. For this reason we call such particles lightly ionizing particles (LIP's). A quark of the standard model also interacts via the strong force and would not be able to penetrate large amounts of material; thus this search is only sensitive to penetrating lightly ionizing particles.

\section{EXPERIMENTAL SETUP}

The MACRO detector is a large $\left(\approx 10000 \mathrm{~m}^{2} \mathrm{sr}\right)$ underground scintillator and streamer tube detector and has been described in detail elsewhere [19,20]. Because of MACRO's large size, fine granularity, high efficiency scintillator, and high-resolution tracking system, it is uniquely suited to look for LIP's. In order to take advantage of this situation a special LIP trigger system has been built.

Using the lowest level energy-based scintillator trigger available in MACRO, it allows a search for particles which interact electromagnetically but deposit much smaller amounts of energy in the scintillator counters than minimum ionizing muons. The inputs are the individual counter lowenergy triggers produced in the PHRASE (one of the gravitational collapse triggers), which have a trigger threshold of about $1.2 \mathrm{MeV}$. Since a typical muon energy loss is about 40 $\mathrm{MeV}$, this trigger threshold allows a search for particles losing less than $1 / 25$ of this.

Streamer tubes are more efficient at triggering on LIP's than the scintillator system. The key to the good sensitivity of the streamer tubes, even to extremely small amounts of ionization, is that even a single ion-electron pair produces a full streamer with reasonable probability.

The measured single ion-pair efficiency for the MACRO tubes, gas mixture, and operating voltage is over 30\%, which is consistent with earlier work [21]. Since selected tracks are required to cross at least 10 streamer tube planes and a LIP trigger only requires that any 6 of them fire, the streamer tube triggering probability is over $99 \%$ for the range of charges considered in this search.

The LIP trigger uses field programmable gate array circuits to form coincidences between counters in the three horizontal planes of MACRO scintillator. The resulting accidental coincidence rate of approximately $10 \mathrm{~Hz}$ would overload the data acquisition and storage system and so it is reduced by requiring a coincidence with at least six streamer tube planes in the bottom part of the detector.

Since a well-reconstructed streamer tube track is required in the final off-line analysis, the streamer tube trigger requirement does not reduce the efficiency of the search, although it reduces accidental coincidences to an acceptable level. The LIP trigger stops the $200 \mathrm{MHz}$ wave-form digitizer (WFD) system and causes the data acquisition system to readout the wave forms of all the counters involved in the trigger.

The use of this trigger allows a physics search for LIP's which is unique in many ways. Some of the main features which distinguish it are as follows.

(1) Sensitivity down to $\frac{1}{5}$ equivalent fractional charge. Previous experiments have only checked for particles with charge $\gtrsim \frac{1}{3}[8]$.

(2) Good acceptance from $\beta=0.25-1.0$. Particles which have a velocity lower than $0.25 c$ are not guaranteed to pass through the detector quickly enough to insure that the LIP trigger will detect a coincidence in the faces of the scintilla- 
tor system. The lowest flux limits for LIP's now come from the very large water Cherenkov detector in Japan (Kamiokande) [22]. However, because of the nature of the Cherenkov process, water detectors are only sensitive to particles with $\beta \geq 0.8$.

(3) Size of detector. The MACRO detector presents $\approx 800$ $\mathrm{m}^{2}$ of fiducial area to downward-going particles. The Cherenkov search at Kamiokande presents a nominal detection area of $130 \mathrm{~m}^{2}$ [22]. The best results from scintillator-based experiments come from even smaller detectors. The search by Kawagoe et al. [23] relied on a detector of only $6.25 \mathrm{~m}^{2}$.

(4) The possibility of searching within large multiple muon bundles for fractional charge. Because of the size and granularity of the MACRO experiment, it is possible to isolate tracks located in muon bundles containing on the order of 20 muons, and to check their energy deposition to see whether they are consistent with LIP's. For both smaller experiments and nongranulated experiments (such as single large volume water experiments like Kamiokande), multiple muon events are rejected from the data sample. If fractionally charged particles were being produced in high energy collisions in the upper atmosphere, previous experiments may have missed the signature due to the particles being buried in the high-multiplicity shower.

(5) Use of high-resolution wave-form digitizers for energy and timing reconstructions. At a trigger threshold of $\approx 1.2 \mathrm{MeV}$ each scintillator counter fires at approximately 2 $\mathrm{kHz}$. The traditional analogue to digital converter (ADC) or time to digital converter (TDC) system is susceptible to errors associated with false starts at this rate (see, for example, [24]). A small pulse triggering the ADC-TDC system just prior to a large pulse can result in partial integration of the large pulse, producing a fake low ionization event.

(6) Use of a high precision limited streamer tube tracking system. Previous underground experiments $[22,23,25]$ did not have independent tracking systems. Since muons that clip the corners of scintillating volumes can be an important source of background, the use of a tracking system is essential for the performance of a low background search. In addition, without a tracking system it is hard to recognize the cases where the actual tracks pass between volumes and accompanying soft $\gamma$ rays enter into the scintillating volumes. This can be a source of background [25]. The use of a tracking system is also one of the reasons that the MACRO can look for fractional charge in high multiplicity muon bundles.

\section{DATA ANALYSIS}

The data for this search comes from two periods. The first ran from July 24th to October 12th of 1995, and the second from December 17th 1995 to November 16th 1996. These were both periods of uninterrupted wave-form and LIP operation with the entire MACRO detector. The live time varied for subsections of the detector and the longest live time was 250 days.

\section{A. Low-energy reconstruction}

Triggering at very low thresholds is challenging. While previous searches have restricted themselves to $\frac{1}{3} e$, this search reaches $\frac{1}{5} e$. For particles with average path lengths through the MACRO scintillator counters the energy deposited is about

$$
40 \mathrm{MeV} \times\left(\frac{1}{5}\right)^{2} \approx 1.6 \mathrm{MeV}
$$

Therefore, in order to be able to reconstruct LIP's which pass through the MACRO, it is necessary to reconstruct energies between 1.5 and $40 \mathrm{MeV}$.

The triggering threshold of the LIP trigger was measured by using muons which passed through small amounts of scintillator in the MACRO detector, and thus deposited small amounts of energy. The measured triggering efficiency is shown in Fig. 1; it is $100 \%$ above $\approx 2 \mathrm{MeV}, 50 \%$ above 1.2 $\mathrm{MeV}$.

Each scintillator counter used in the analysis was calibrated using naturally occurring low-energy $\gamma$ rays. The most important of these $\gamma$ rays for the calibration is the $2.6 \mathrm{MeV}$ line from the radioactive decay chain:

$$
\begin{aligned}
& { }_{81}^{208} \mathrm{Tl} \\
& \longrightarrow{ }_{82}^{208} \mathrm{~Pb}^{*}+\beta^{-}+\overline{\nu_{e}} \\
& \qquad{ }_{{ }_{82}^{208} \mathrm{~Pb}+\gamma(2.6 \mathrm{MeV})}
\end{aligned}
$$

After every event which causes a readout of the WFDs, one millisecond worth of WFD data is collected for every counter involved in the event. For fast particles such as muons only the first few microseconds of the WFD data is relevant. The rest of the data is recorded in order to search for slowly moving particles such as magnetic monopoles. The one millisecond of data contains small pulses caused by naturally occurring radioactivity. By looking at these radioactivity pulses we can reconstruct the low energy spectrum. Figure 2 shows this spectrum for one of the MACRO scintillator counters.

The solid line is a fit to a falling radioactivity spectrum plus two Gaussians, one associated with the $2.6 \mathrm{MeV}{ }_{81}^{208} \mathrm{Tl}$ line, and the other, with the $1.4 \mathrm{MeV}^{40} \mathrm{~K}$ line. A full GEANT Monte Carlo Step was performed to determine where the absolute energies of the lines in this spectrum should be, and the information from the fit is used to make a calibration constant to convert between observed photomultiplier tube (PMT) signal measured in the wave forms and deposited energy.

Since $1-5 \mathrm{MeV}$ is the important signal region for the LIP search, reconstructing the low-energy spectrum in this region is proof that we can also reconstruct LIP's in this region. For this reason, we require a counter to have a good calibration in order to use it for the LIP analysis. Aside from a very few nonfunctional scintillator counters, in practice, what this means is that only the counters placed in three horizontal planes were used, and the counters in vertical planes were not.

\section{B. Time reconstruction}

It is important to determine an event's longitudinal position in a counter from its WFD data. Calibration events as 


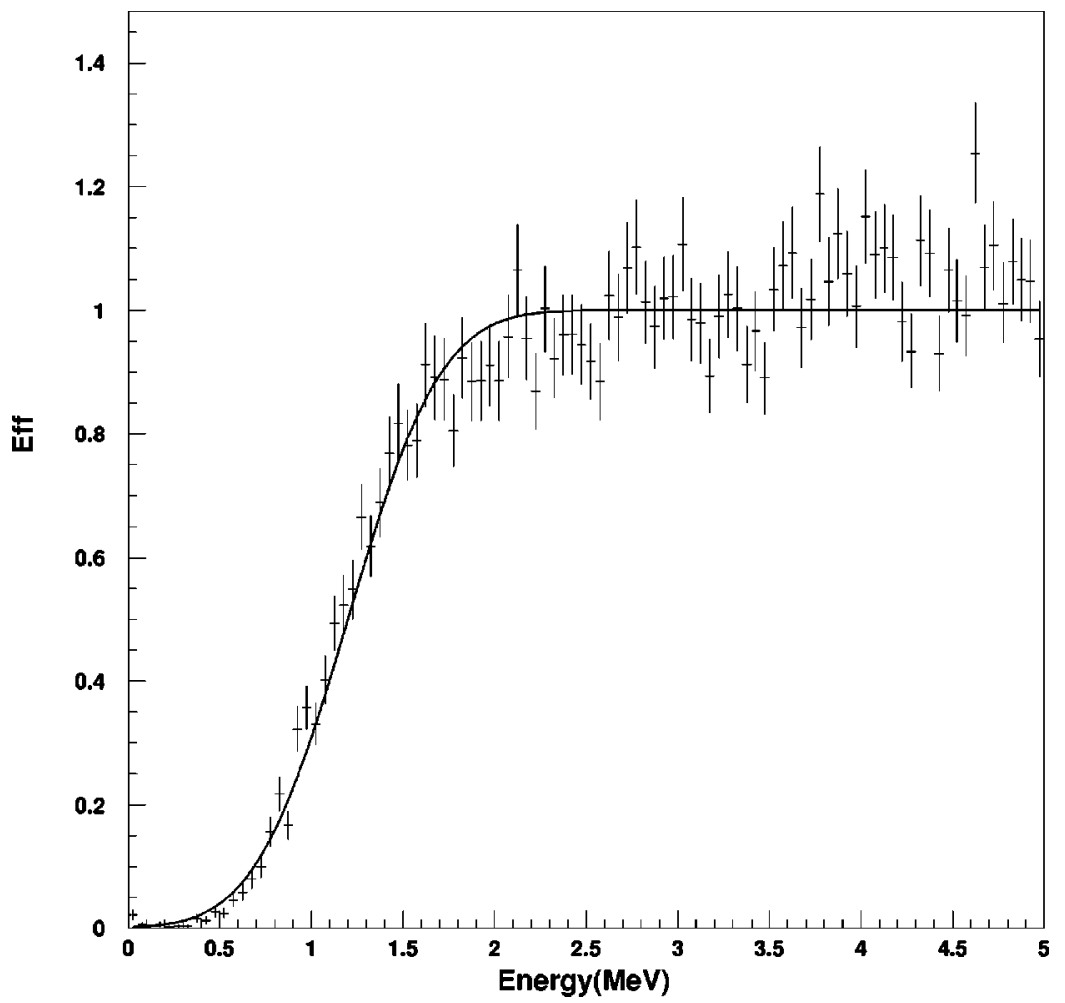

FIG. 1. The measured efficiency of triggering the low-energy PHRASE trigger and the LIP trigger as a function of energy. Some measured efficiencies are greater than $100 \%$ because the normalization factor used is only an estimate of the true normalization as a function of energy.

described in Sec. III A have no associated streamer tube track, and so this is the only source of the information necessary to correct for the light attenuation of the scintillator. For particles passing through the detector, we require consis- tency between the longitudinal position of the event independently determined by the streamer tubes and the PMT signals. This reduces the background due to accidental coincidences between a small radioactivity pulse somewhere

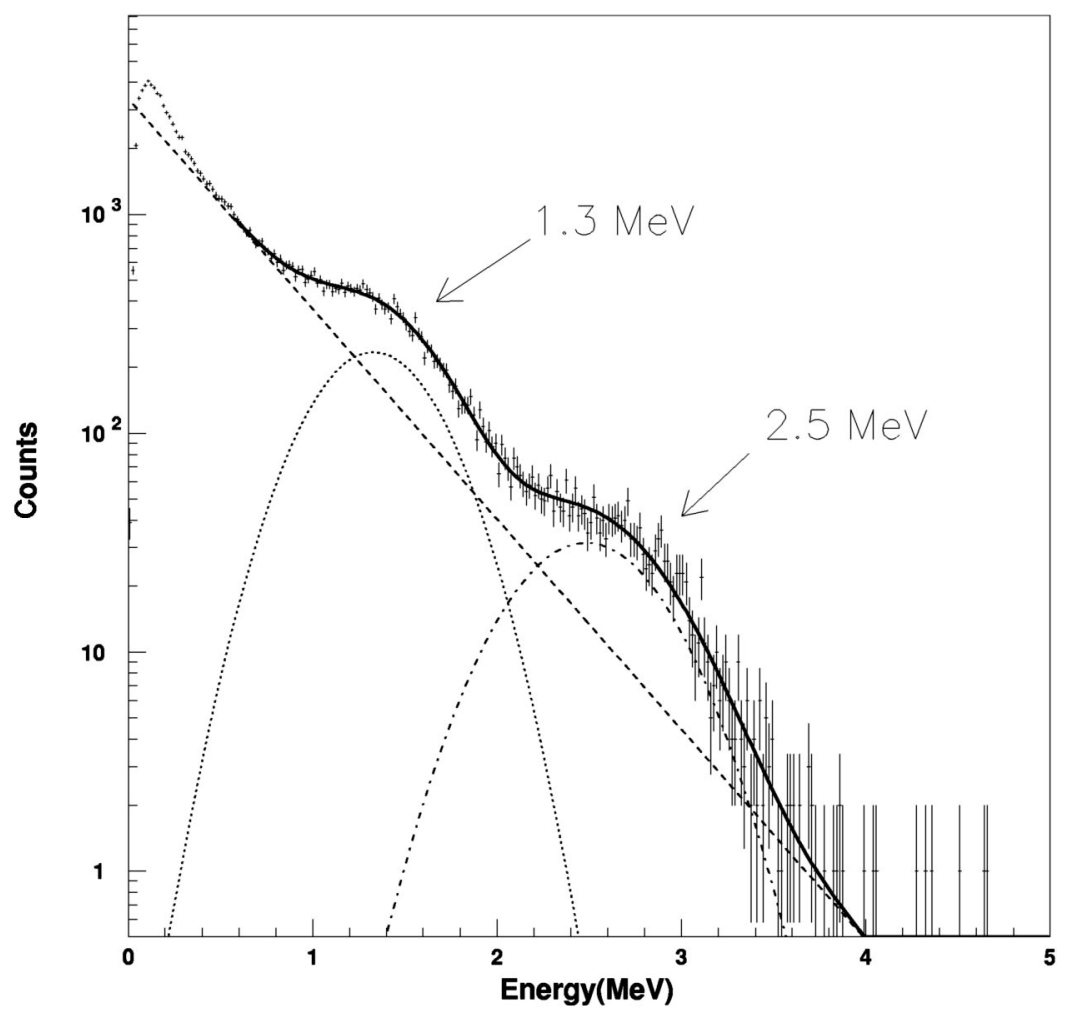

FIG. 2. A fit to low-energy WFD data with a falling radioactive spectrum, and a Gaussian associated with both the $2.6 \mathrm{MeV} \gamma(\mathrm{T} 1)$ and 1.4 $\mathrm{MeV} \gamma(\mathrm{K})$ line. Each energy bin is $16.7 \mathrm{keV}$ wide. The eight parameters of the fit are the normalization and slope for an exponential and the normalization, mean, and width for the two Gaussians. 


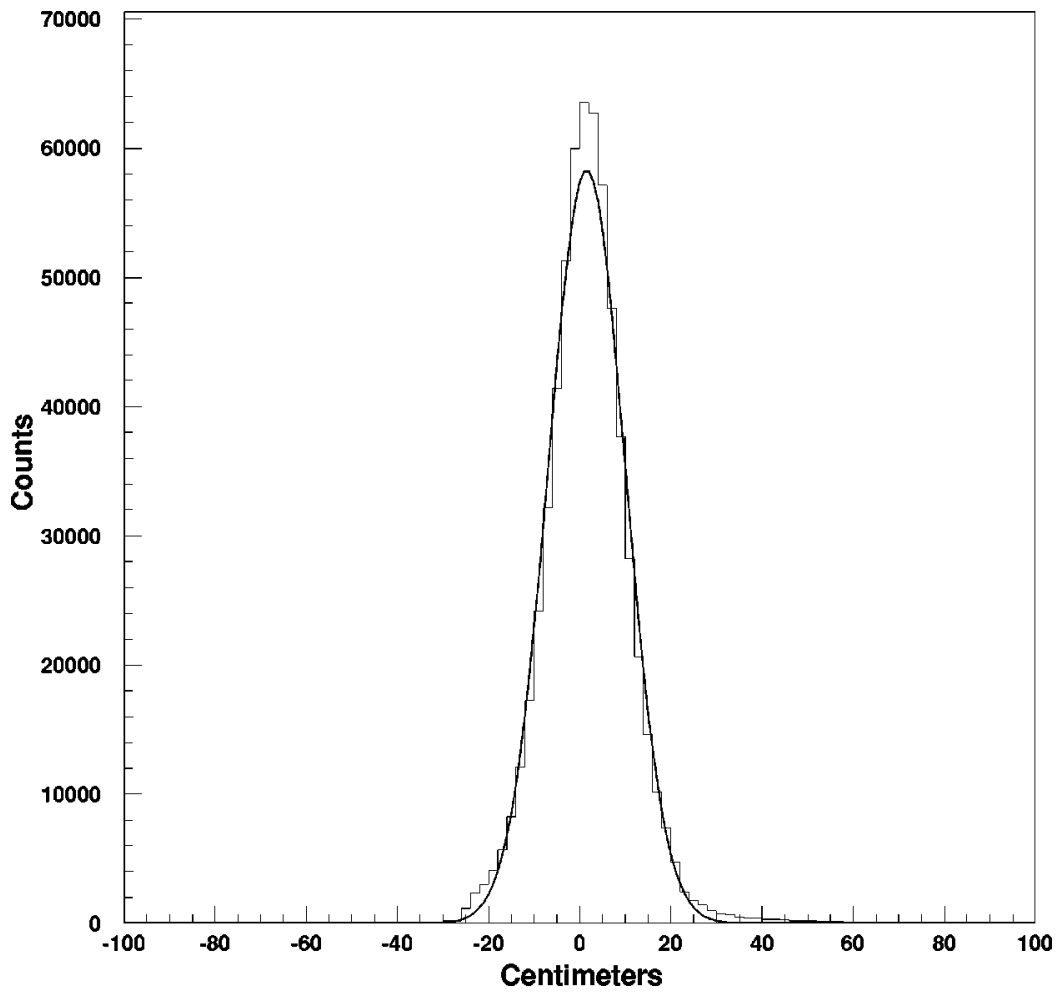

in the counter followed by a muon passing through a crack in the detector. The width of the position resolution determines how tightly this cut can be made.

The longitudinal position in a counter of an event can be calculated using the WFD information with the expression:

$$
\operatorname{pos}=\frac{\Delta t \times v}{2},
$$

where $\Delta t$ is the difference in time between the pulses on the two sides of the counter (as measured by the wave forms), and $v$ is the effective speed of light in the counter. Figure 3 shows the difference between the positions of muons passing through a scintillator counter calculated by the streamer tube tracking system and that calculated by the WFD system for all of the scintillator counters used in the analysis.

These timing results were obtained by first performing a software simulation of a constant fraction trigger [26] to obtain an initial estimate of the longitudinal position. This circuit triggers at the point on the leading edge of a pulse which is a fixed fraction of the maximum height of the pulse. In order to estimate at what time the pulse crosses the fixed fraction of the maximum peak voltage $(20 \%$ was used for this analysis) a simple linear fit was used between the two samples closest to the point of crossing.

A neural network was then used to further refine the estimate of the longitudinal position. The neural network was trained with a sample of events using the position obtained from the streamer tubes. We chose to use a neural network since we did not find an alternative which provided the same or better precision and was less computationally intensive. A more detailed description of the network used can be found in [18].
FIG. 3. Difference in position calculated by the streamer tubes and that by the PMT signals for a sample of the muon data. The rms deviation from the mean (sigma) is $8.5 \mathrm{~cm}$. All counters used in the analysis are included in this histogram; individual counters have smaller sigmas.

\section{SEARCH RESULTS}

After calibration, the data set was examined for LIP's in both single and multiple track events. In order to be considered in the analysis, an event had to satisfy three requirements: the LIP trigger must have fired; at least one track must have been reconstructed in the streamer tube system; and finally, at least one of the reconstructed tracks must have passed through counters in the top, center, and bottom of the detector. There were approximately 1.3 million events which satisfied these requirements. The data set was broken into two exclusive pieces, a single track and a multiple track set, with approximately $90 \%$ of the events being in the single track sample.

Each of the selected events was then examined to determine its rate of energy loss in the scintillator. For each of the counters that a selected particle passed through, the reconstructed energy was scaled to a common path length of 19 $\mathrm{cm}$, the distance a vertical muon passing through a scintillator counter traveled. To reduce the chance that anomalies would affect the result, the maximum energy in any of the counters was used as a measure of the particle's energy loss. Figure 4 is a histogram of this distribution for all of the tracks (in both the single and multiple track sample) that passed the selection criteria.

The trigger becomes more than $60 \%$ efficient at about 1.2 $\mathrm{MeV}$ and quickly rises to $100 \%$ efficiency. Then, at about 20 $\mathrm{MeV}$, the efficiency of this search quickly drops to zero because a cut must be made to reject muons. Before any cuts, there are events in the region where LIP's would be expected to appear $(\$ 20 \mathrm{MeV})$. These result from two classes of reconstruction errors. First, there are cases where tracks passed close to the edge of a scintillator counter or very close to a 


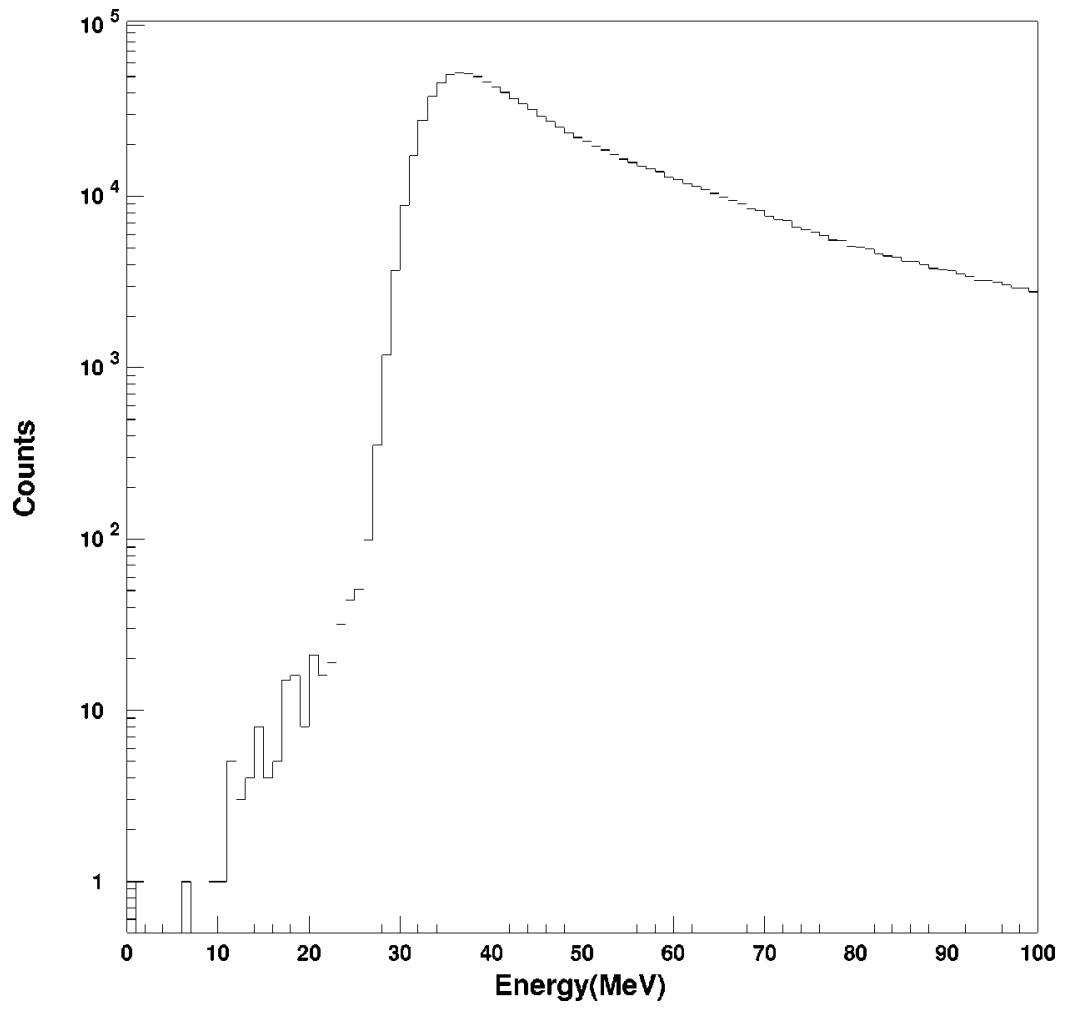

FIG. 4. The maximum energy reconstructed in any counter on the track. Only an event in which every counter has a low energy will show up as having low energy in this histogram. The reconstructed energy has been scaled to a $19 \mathrm{~cm}$ path length for all events.

phototube and the energy was incorrectly reconstructed. We, therefore, also exclude tracks which at their center in the scintillator volume are located in the final $10 \mathrm{~cm}$ of a scintillator counter. By requiring that all tanks hit by the track have this fiducial requirement, the number of events in the single track sample is reduced by $\approx 4 \%$.

Second, there are events in which the position recon- structed by timing in the scintillator counter is inconsistent with that obtained by the streamer tube tracking system, possibly due to random noise in the streamer tubes confusing the tracking algorithm. We require that the position of the particle passage as reconstructed in the streamer tubes agrees with the position as reconstructed by the neural network timing procedure to within $\pm 45 \mathrm{~cm}$, which is about $3 \sigma$ for

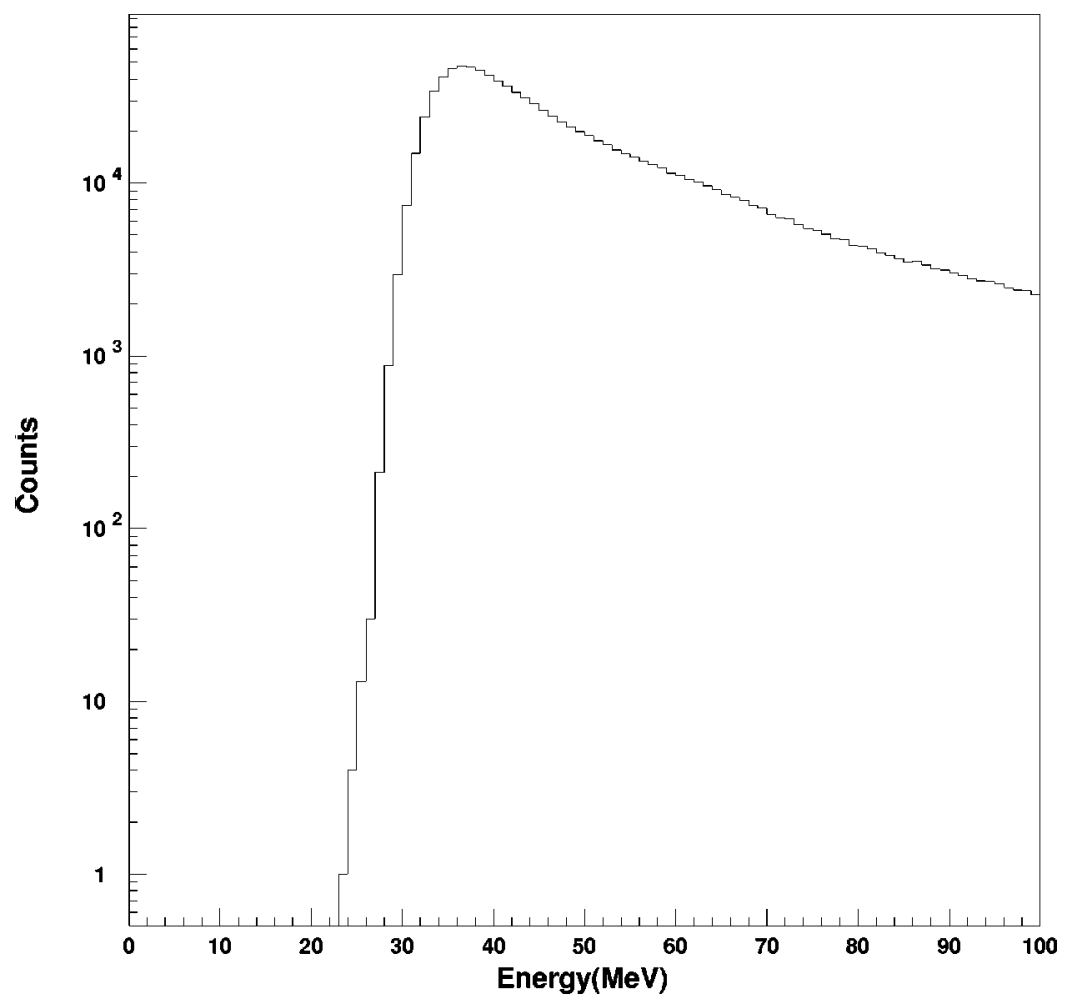

FIG. 5. The maximum energy reconstructed in any counter on the track for events in the single track sample. The streamer tube and scintillator position reconstruction have been required to agree to within $\pm 45 \mathrm{~cm}$, and fiducial cuts in the scintillator volume have been applied. 


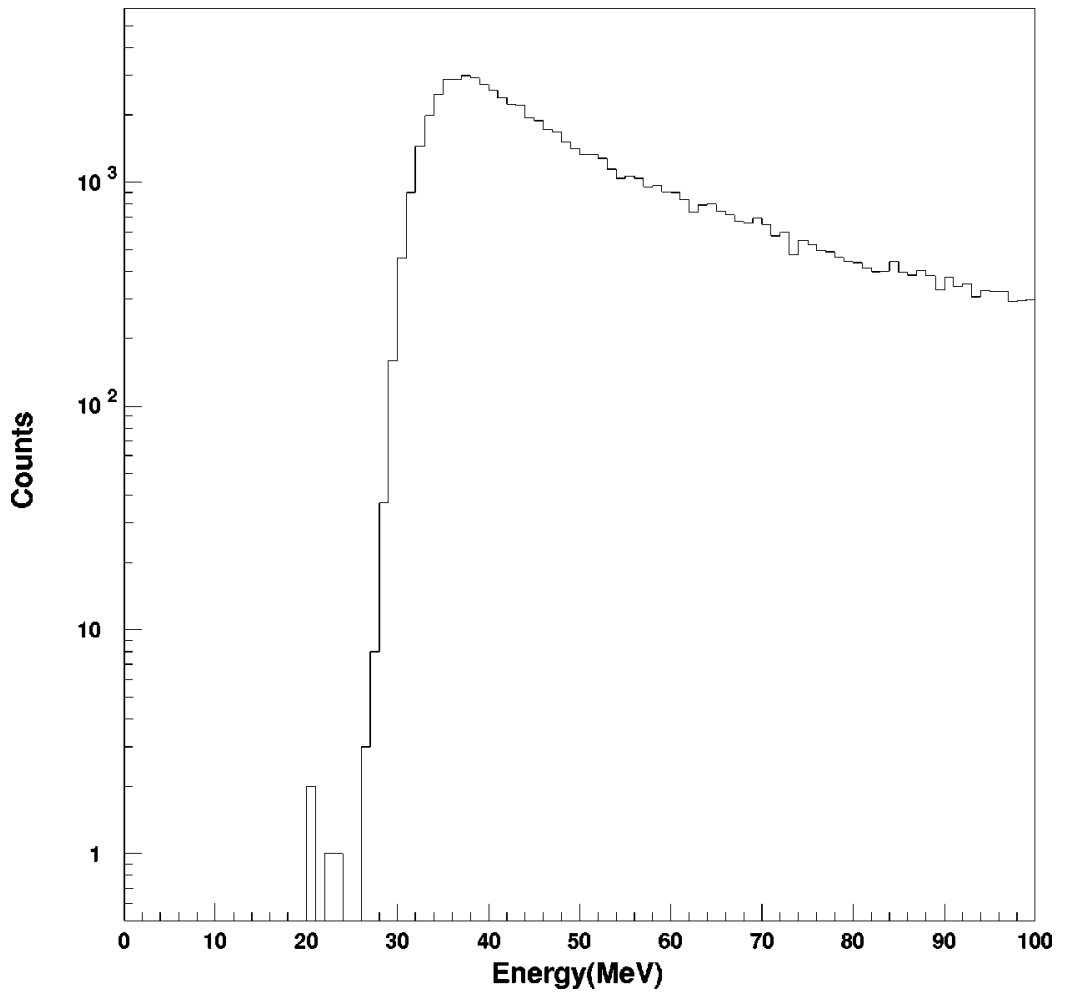

FIG. 6. The maximum energy reconstructed in any counter on the track of the event for the multiple track sample. The streamer tube and scintillator position reconstruction have been required to agree to within $\pm 45 \mathrm{~cm}$, and fiducial cuts in the scintillator volume have been applied. The events with the three lowest energies arose from falsely reconstructed tracks in the streamer tube system. There are no real tracks associated with these events. energy depositions smaller than $5 \mathrm{MeV}$. This cut removes $1.8 \%$ of the data.

Figure 5 is the distribution of the maximum counter energy on a track for all of the single muon tracks considered in the analysis after the fiducial and position agreement cut. The expected signal region for LIP's is below $20 \mathrm{MeV}$.

Figure 6 is the same distribution for the multiple track sample. There are four events in the multiple tracks sample with maximum deposited energies between 20 and $23 \mathrm{MeV}$. The minimum entry in the distribution for the single track sample is $23 \mathrm{MeV}$. These four events were examined by hand. All four were reconstructed as double muons by the tracking algorithm. In three cases, the tracking algorithm failed and assigned a track where one really did not exist. This nonexistent track intersected counters that were actually hit, but the calculated path lengths with the fake track were incorrect. The fourth event had a maximum energy loss of 23 $\mathrm{MeV}$. This event shows no anomalies and is consistent with the lowest energy seen in the single track sample.

\section{CONCLUSIONS}

In the approximately one year of running that this search covers, no candidates for LIP's were observed. This search was sensitive to particles with charges greater than $\frac{1}{5} e$ and $\beta$ between approximately 0.25 and 1.0 . Unlike previous experiments, this search attempted to find LIP's in both single track events and buried among the tracks of multiple muon showers.

For the single track sample, the assumption of an isotropic flux yields a $90 \%$ C.L. upper flux limit of $\Phi \leqslant 9.2$ $\times 10^{-15} \mathrm{~cm}^{-2} \mathrm{~s}^{-1} \mathrm{sr}^{-1}$.

Once again, it should be emphasized that the energy loss considered for particles in this search is due solely to atomic excitation and ionization. If LIP's are present in the cosmic rays and they interact strongly as well as electromagnetically they will not be able to travel through enough rock to reach the MACRO detector before they interact strongly. Only if strongly interacting LIP's were produced in the rock very

TABLE I. A summary of limits in LIP searches expressed in units of $\mathrm{cm}^{-2} \mathrm{~s}^{-1} \mathrm{sr}^{-1}$. This limit (MACRO) is compared with limits from the water Cherenkov Kamiokande experiment and the scintillator-based LSD experiment. The MACRO experiment is alone in setting a limit on $\frac{1}{5} e$ and $\frac{1}{4} e$ charged particles. A ".' in the table means that the listed experiment was not sensitive to the relevant charge while "Not quoted" means that while in principle the detector was sensitive, the authors chose not to report a limit for that charge.

\begin{tabular}{cccccc}
\hline \hline & & \multicolumn{5}{c}{ Charge } \\
Search & $\frac{1}{5}$ & $\frac{1}{4}$ & $\frac{1}{3}$ & $\frac{1}{2}$ & $\frac{2}{3}$ \\
\hline This search & $2.8 \times 10^{-14}$ & $1.0 \times 10^{-14}$ & $9.5 \times 10^{-15}$ & $1.1 \times 10^{-14}$ & $2.0 \times 10^{-14}$ \\
LSD & - & - & $2.3 \times 10^{-13}$ & Not quoted & $2.7 \times 10^{-13}$ \\
Kamiokande & - & - & $2.1 \times 10^{-15}$ & Not quoted & $2.3 \times 10^{-15}$ \\
\hline \hline
\end{tabular}




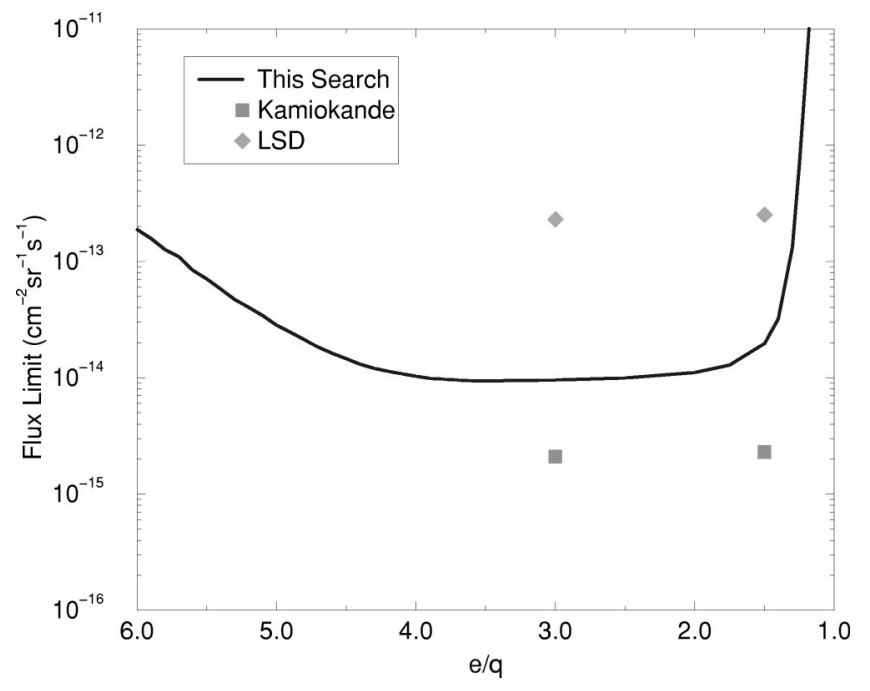

FIG. 7. The upper limit on LIP fluxes at $90 \%$ confidence level established by this search. $q$ is the charge of the LIP. Also shown are the limits from the searches done at the Kamiokande and LSD experiments. Unlike those experiments we report a limit for a continuous range of charges. For this analysis the stated flux limits are valid for a $\beta$ range of $0.25-1.0$.

near the detector would this search be sensitive to them.

The two best experiments to compare this result with are the liquid scintillator detector (LSD) experiment [25] and the Kamiokande experiment [22]. While LSD had the best scintillator-based limit in the world prior to this experiment, Kamiokande has the lowest limit. Both of these experiments only claim sensitivity to $\frac{1}{3} e$ and $\frac{2}{3} e$ charged particles.

Table I summarizes the limits of this search in comparison to other searches. For the entries marked "Not quoted," the experiments do not report a limit for that charge although the experiment should have been sensitive to that energy deposition. At least in the case of LSD there were two candidates in the $\frac{1}{2} e$ region which were ignored because they were not considering $\frac{1}{2} e$ charged particles. In the Kamiokande experiment only $\frac{1}{3} e$ and $\frac{2}{3} e$ were searched for.

Unlike the other two searches this search is sensitive to a continuous range of charges from $\frac{1}{5} e$ to close to the charge of an electron. This limit is shown in Fig. 7. This search had no candidates and required hand scanning of only 3 in 1.2 million events.

In order to compare flux limits for LIP's from different experiments one must keep several factors in mind. First of all, this is a limit on the flux of local LIP's at the site of the detector. Different mechanisms for LIP production result in different properties for their flux. One possibility is that the LIP particles are produced very close to the detector by some unknown neutral particle or mechanism. In this case, one could indeed expect a location independent, isotropic flux.

However, for the more general case of LIP production far away from the detector, one expects different fluxes of LIP particles in different underground locations. At each detector site there will be a unique and nontrivial angular distribution, because of different rock thickness above the detectors. This will be true if the LIP particles are produced near the detector in high-energy muon showers, in cosmic ray showers in the atmosphere, or if they are impinging on the Earth from outer space.

Note that only particles above some minimum energy can reach an underground detector from the atmosphere, because of the ionization loss in the Earth. For the case of MACRO, which has a minimum depth of 3300 meters of water equivalent, the initial energy of a $\frac{1}{5} e$ charged particle before it enters the earth must be $\geqslant 20 \mathrm{GeV}$. In comparison, the Kamiokande experiment has an overburden of 2700 meters of water equivalent, and the LSD experiment is covered with 5000 meters of water equivalent so the energy thresholds should be correspondingly lower and higher, respectively.

In a general discussion such as this one we can only make some qualitative remarks. If the LIP particles are produced in the atmosphere they should not arrive from directions below the horizon. A $\frac{1}{5} e$ charged particle would travel 25 times as far as a muon by virtue of its reduced energy loss, but that distance is still very small compared to the diameter of the earth.

To compare the results of the different experiments one should therefore, in principle, consider a particular physical model of production of the particles, a detailed description of the material above the detectors, and the detector acceptances (including their angular dependences).

\section{ACKNOWLEDGMENTS}

We gratefully acknowledge the support of the director and of the staff of the Laboratori Nazionali del Gran Sasso and the invaluable assistance of the technical staff of the Institutions participating in the experiment. We thank the Istituto Nazionale di Fisica Nucleare (INFN), the U.S. Department of Energy and the U.S. National Science Foundation for their generous support of the MACRO experiment. We thank INFN, ICTP (Trieste), and NATO for providing financial support (FAI) for non-Italian citizens.
[1] R. Millikan, Philos. Mag. 19, 209 (1910).

[2] E. D. Bloom et al., Phys. Rev. Lett. 23, 930 (1969).

[3] R. P. Feynman, Phys. Rev. Lett. 23, 1415 (1969).

[4] M. Gell-Mann, Phys. Lett. 8, 214 (1964).

[5] G. Zweig, CERN Report No. 8182/TH401 (1964).

[6] G. Zweig, CERN Report No. 8419/TH412 (1964).

[7] P. Smith, Annu. Rev. Nucl. Part. Sci. 39, 73 (1989).

[8] L. Lyons, Phys. Rep. 129, 225 (1985).
[9] H. V. Klapdor-Kleingrothaus and A. Staudt, Non Accelerator Particle Physics (IOP, London, 1995).

[10] L. W. Jones, Rev. Mod. Phys. 49, 717 (1977).

[11] V. Halyo et al., Phys. Rev. Lett. 84, 2576 (2000).

[12] P. H. Frampton and T. Kephart, Phys. Rev. Lett. 49, 1310 (1982).

[13] S. M. Barr, D. B. Reiss, and A. Zee, Phys. Rev. Lett. 50, 317 (1983). 
[14] H.-W. Yu, Phys. Lett. 142B, 42 (1984).

[15] K. Yamamoto, Phys. Lett. 120B, 157 (1983).

[16] Fang-Xiao Dong, Pei-You Xue, Tung-Sheng Tu, and XianJian Zhou, Phys. Lett. 129B, 405 (1983).

[17] A. De Rújula, R. Giles, and R. Jaffe, Phys. Rev. D 17, 285 (1978).

[18] C. W. Walter, Ph.D. thesis, California Institute of Technology, 1997.

[19] S. Ahlen et al., Nucl. Instrum. Methods Phys. Res. A 324, 337 (1993).

[20] M. Ambrosio et al., Astropart. Phys. 4, 33 (1995).
[21] G. Battistoni et al., Nucl. Instrum. Methods Phys. Res. A 235, 91 (1985).

[22] Kamiokande-II Collaboration, M. Mori et al., Phys. Rev. D 43, 2843 (1991).

[23] K. Kawagoe et al., Lett. Nuovo Cimento Soc. Ital. Fis. 41, 604 (1985).

[24] J. Napolitano et al., Phys. Rev. D 25, 2837 (1982).

[25] M. Aglietta et al., Astropart. Phys. 2, 29 (1994).

[26] W. Leo, Techniques for Nuclear and Particle Physics Experiments (Springer-Verlag, Heidelberg, 1987). 